\title{
Thickness dependence of the ground-state properties of thin films of the heavy-fermion compound $\mathrm{CeCu}_{6}$
}

\author{
D. Groten, G. J. C. van Baarle, J. Aarts, G. J. Nieuwenhuys, and J. A. Mydosh \\ Kamerlingh Onnes Laboratory, Leiden University, 2300RA Leiden, The Netherlands
}

(Received 21 March 2001; revised manuscript received 25 June 2001; published 21 September 2001)

\begin{abstract}
High-quality thin polycrystalline films of the heavy-fermion compound $\mathrm{CeCu}_{6}$ were prepared by sputter deposition. The thicker of these films (with thickness up to around $200 \mathrm{~nm}$ ) reproduce the properties of the bulk compound $\mathrm{CeCu}_{6}$. As the thickness of the films is decreased, our measurements display strong deviations from the bulk properties, namely, a suppression of the heavy-fermion state. We show that possible "external" effects, such as disorder, oxidation and morphology can be excluded and that this size effect is therefore an intrinsic property of $\mathrm{CeCu}_{6}$. In addition, we investigate possible scenarios explaining the size effect, and find that the proximity of $\mathrm{CeCu}_{6}$ to a quantum phase transition can account for this striking result.
\end{abstract}

DOI: 10.1103/PhysRevB.64.144425

PACS number(s): 75.30.Mb, 71.10.Ay, 71.27.+a

\section{INTRODUCTION}

Strongly correlated electron systems have been studied extensively over the past 2 decades and produced a plethora of new and intriguing results. Among these systems are exotic heavy-fermion superconductors, ${ }^{1,2}$ heavy-fermion paramagnets, ferromagnets or antiferromagnets with tiny moments, ${ }^{3,4}$ spin glasses, ${ }^{5}$ Kondo insulators, ${ }^{6}$ and non-Fermi liquids. ${ }^{7}$ Heavy-fermion compounds are often referred to as the "concentrated limit" of dilute Kondo alloys, because the local spin fluctuations arising from the hybridization of the $f$ electrons with conduction-band states have essentially the same origin as has the single-impurity Kondo effect. The Kondo-lattice concept can consistently explain many of the bulk properties of Ce-based heavy-fermion compounds, in particular, the formation of a narrow coherent heavy-fermion band with $f$ symmetry at low temperatures. ${ }^{8}$ It also provides a single scaling temperature $T^{*}$ characterizing the properties of the system at low temperatures. Now an intriguing question arises: What length scales should be associated with (i) the coherent quasiparticle state and (ii) the correlations arising from the single-impurity Kondo effect? In other words, will a heavy-fermion system keep its bulk properties when one of more dimensions are reduced?

In this paper, we address these queries by studying the transport properties of thin films of $\mathrm{CeCu}_{6}$ as a function of their thickness. $\mathrm{CeCu}_{6}$ is an archetypal heavy-fermion system with one of the largest-known electronic-specific-heat coefficients $^{9,10}$ and no magnetic or superconducting transition down to $20 \mathrm{mK} .{ }^{11-13}$ This makes it an ideal system to study the ground-state properties of the heavy-fermion state. Furthermore, it has attracted great attention in the past few years due to its proximity to an antiferromagnetic quantum critical point (QCP). When doping with gold $\left(\mathrm{CeCu}_{6-x} \mathrm{Au}_{x}\right)$ non-Fermi-liquid behavior is observed in $\mathrm{CeCu}_{5.9} \mathrm{Au}_{0.1}$ and an antiferromagnetic ground state is found for $x>0.1{ }^{14}$ Our main result is that as the thickness of the films is decreased, the heavy Fermi-liquid state is suppressed, and the behavior becomes that of a dilute Kondo-impurity system. This result can best be explained with an increase of the characteristic temperature scale $T^{*}$ as the thickness of the films is de- creased. We argue that the proximity of $\mathrm{CeCu}_{6}$ to the QCP can account for a correlation length of the quantum fluctuations of ca. $10 \mathrm{~nm}$, which leads to the increase of $T^{*}$ as the thickness of the films approaches this length.

\section{EXPERIMENT}

The thin films of $\mathrm{CeCu}_{6}$, with thicknesses ranging from around $10 \mathrm{~nm}$ to around $200 \mathrm{~nm}$, were prepared by cosputtering under ultrahigh vacuum conditions from two targets of pure $\mathrm{Cu}(99.99 \%)$ and $\mathrm{Ce}$ (Ames Laboratory, 99.995\%). They were deposited onto $\mathrm{Si}(100)$ substrates with a $\mathrm{Si}_{3} \mathrm{~N}_{4}$ buffer layer at a temperature of $350^{\circ} \mathrm{C}$, and subsequently covered in situ at room temperature against oxidation with a Si layer. Thickness and composition of the films were determined by electron-probe microanalysis and Rutherford backscattering (RBS). The films are found to be homogeneous both in plane and as a function of depth, and have sharp interfaces with substrate and protection layer. X-ray diffraction (XRD) measurements demonstrate that the films are polycrystalline (orthorhombic structure) with lattice parameters within $0.1 \%$ of those found for bulk samples, ${ }^{15}$ and show a slightly preferred orientation of the $b$ axis in growth direction. As the thickness of the films is reduced, no variations of the lattice constants, nor of the preferred orientation, are observed. High-resolution electron microscopy (HREM) has enabled us to prove that the grain size is the same in films of thickness $189 \mathrm{~nm}$ and $53 \mathrm{~nm}$, and of the order of 10 $\mathrm{nm}$, independent of film thickness. Also, our RBS measurements have enabled us to put an upper limit of 2 at. \% on oxygen content. Resistance measurements were performed on structured samples in a four-probe geometry (50-200 $\mu \mathrm{m}$ wide and $2-3 \mathrm{~mm}$ between the voltage contacts) in applied magnetic fields up to $8 \mathrm{~T}$ and at temperatures down to 20 $\mathrm{mK}$. We have shown in an earlier paper ${ }^{15}$ that for a film of thickness $189 \mathrm{~nm}$, the measurements of resistance vs temperature and those of the magnetoresistance mimic the properties of bulk samples, establishing the formation of a heavy Fermi-liquid state at low temperatures in such thick films.

The resistivity data in zero magnetic field as a function of 


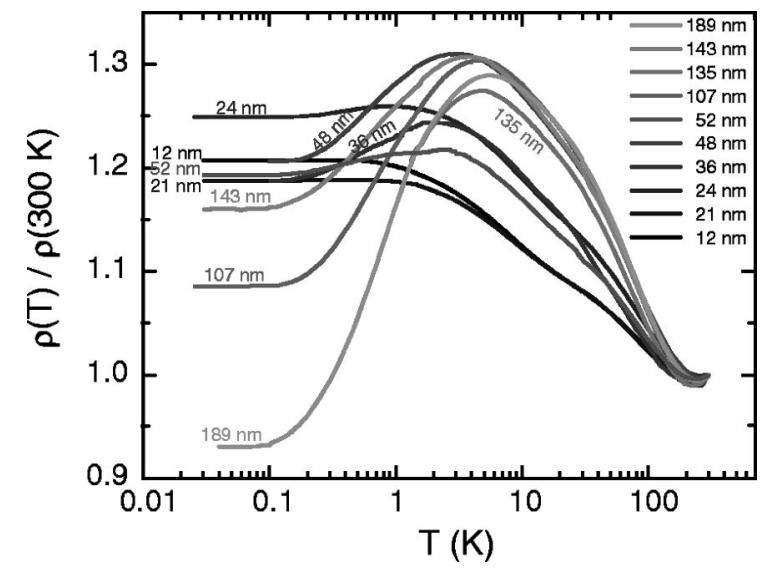

FIG. 1. Normalized resistivity $\rho / \rho(T=300 \mathrm{~K})$ as a function of temperature $T$ on a logarithmic scale in zero magnetic field for $\mathrm{CeCu}_{6}$ films of varying thickness. A darker line means a thinner film.

temperature is shown for samples of various thicknesses in Fig. 1. The absolute values were normalized to those measured at room temperature. This allows a better comparison of the different samples, by removing uncertainties due to the determination of the thickness $( \pm 10 \%)$. Moreover, we are not able to subtract nonmagnetic contributions to the resistivity, such as phonon contributions-this would require the fabrication of $\mathrm{LaCu}_{6}$ thin films. At high temperatures, the logarithmic temperature scale enables us to compare these results to a dilute Kondo system, in which the temperature dependence of the resistivity is $-\ln T$. There is no clear range of temperatures for any sample in which this logarithmic behavior is clearly observed. Crystalline electric field (CEF) effects complicate the simple Kondo picture at temperatures of the order of $50 \mathrm{~K}$, due to the occupancy of higher-lying CEF levels. ${ }^{16}$ The general tendency at high temperatures is that the slope $s=-d \rho / d(\ln T)$ is lower for thinner films than for thicker films.

At low temperatures, we see that large differences are found between the thick films and the thin films. Whereas for the thick films, there is a clear maximum of the resistivity $\rho$ at a temperature $T_{\max }$ followed by a large drop upon lowering the temperature, in contrast, the resistivity just saturates for the thinnest films. The absolute values of the residual resistivity $\rho_{0}$ do not show any clear thickness dependence. They are shown as a function of film thickness $d$ in Fig. 2 (right axis). The black bar represents the approximate range of values found in literature-averaged over the three crystallographic directions for single crystals. Two remarks can be made based on these measurements: (i) The dependence of the residual resistivity on the thickness is very weak, proving that no additional disorder is induced when the samples are made thinner. (ii) The values of the residual resistivity clearly lie in a metallic regime, well below the Ioffe-Regel limit where the mean free path $l$ is equal to the interatomic distance, which for $\mathrm{CeCu}_{6}$ would give a maximum resistivity of $300 \mu \Omega \mathrm{cm}$ for a metallic state. Also in Fig. 2 the values of $T_{\max }$ as a function of thickness (gray symbols, left axis) are shown. The bulk values found in the literature are as high as $15 \mathrm{~K}$ for particular crystallographic directions. A clear

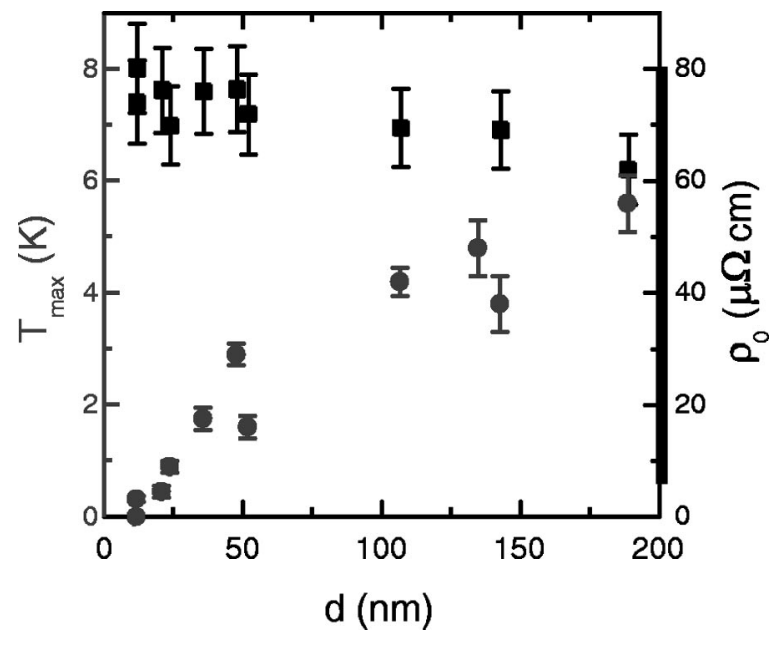

FIG. 2. Black squares, right axis: Residual resistivity $\rho_{0}$ of thin films of $\mathrm{CeCu}_{6}$ measured at $\sim 30 \mathrm{mK}$ as a function of film thickness $d$. The black bar on the right axis represents the range of literature values found for bulk samples (resistivities for the three crystallographic directions were averaged for single crystals). Gray circles, left axis: Values of $T_{\max }$ as a function of film thickness.

tendency toward suppression of $T_{\max }$ is found as the thickness of the films is decreased.

For $d>20 \mathrm{~nm}$, Fermi-liquid behavior is found ${ }^{15}$ at very low temperatures $(T<200 \mathrm{mK}), \rho=\rho_{0}+A T^{2}$, where $A$ is the Fermi-liquid coefficient related to the effective mass $m^{*}$ of the quasiparticles by $A \propto\left(m^{*}\right)^{2} .{ }^{17}$ The values obtained for $A$ are shown in Fig. 3 as a function of film thickness. For comparison, the values found in literature for bulk samples are symbolized by the gray bar on the right side of the figure. There is a dramatic decrease of $A$ as the thickness of the

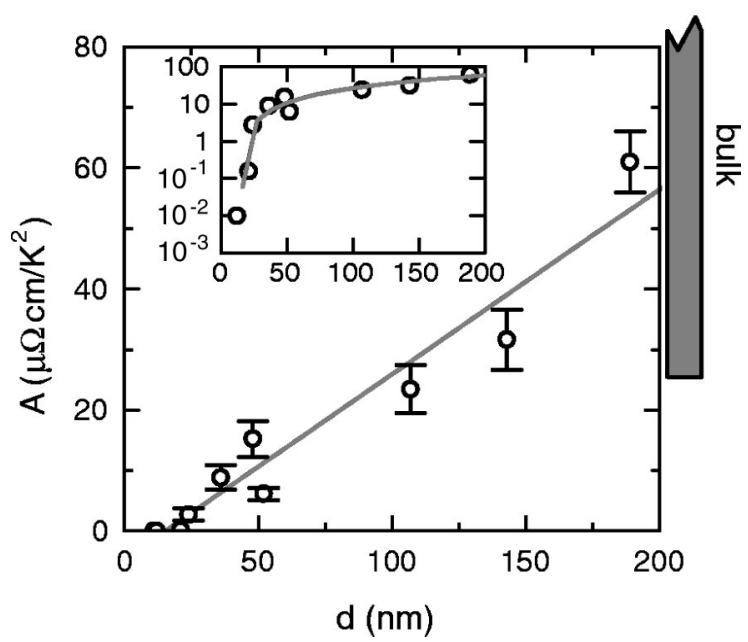

FIG. 3. Fermi-liquid coefficient $A$ of the low-temperature resistivity as a function of sample thickness. The inset shows the same points on a logarithmic scale. The gray bar signifies the range of literature values for $A$ found for bulk samples. For clarity reasons the bar is not extended to the maximum value of $112 \mu \Omega \mathrm{cm} / \mathrm{K}^{2}$. For single crystals, values along the three crystal directions have been averaged. The solid gray line is a linear fit to the data. A extrapolates to zero at $\approx 15 \mathrm{~nm}$. 


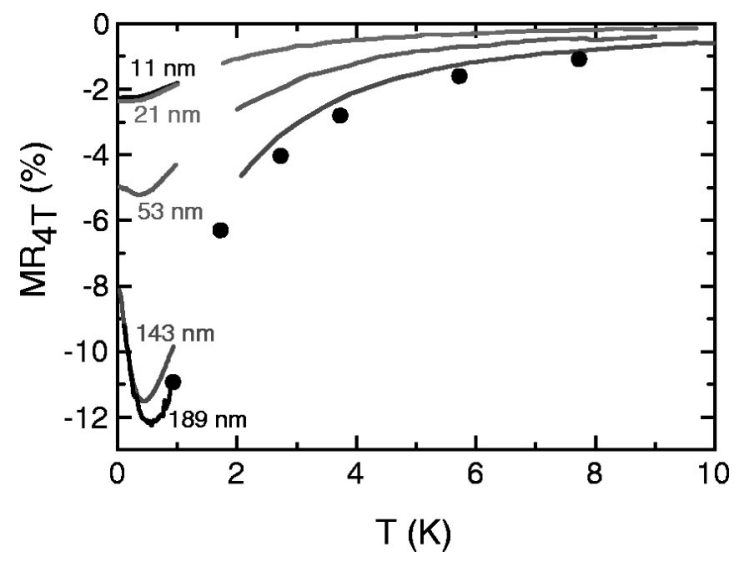

FIG. 4. Magnetoresistance MR measured in a field of $4 \mathrm{~T}$ as a function of temperature for $\mathrm{CeCu}_{6}$ thin films of varying thickness. Only a few measurement points were taken for the film of thickness $189 \mathrm{~nm}$, represented by the black dots.

samples is reduced. Below $50 \mathrm{~nm}$ this drop is better viewed on a logarithmic scale, as seen in the inset.

Overall, the value of $A$ decreases by nearly four orders of magnitude, which should be contrasted with a maximum difference by a factor of 5 between the different bulk samples. For the thinnest films of $11 \mathrm{~nm}$ and $12 \mathrm{~nm}$, the resistivity just saturates down to lowest temperatures, and no value of $A$ can be defined. Our results clearly demonstrate that the heavy Fermi-liquid state is suppressed in our thinnest films.

The magnetoresistance (MR) is also strongly thickness dependent: The magnitude of the negative MR decreases as the thickness is decreased for all temperatures below $10 \mathrm{~K}$. This is shown in Fig. 4 where measurements of the MR in an applied field of $4 \mathrm{~T}$ were performed. The MR is defined as $\mathrm{MR}=[\rho(H)-\rho(0)] / \rho(0)$. The thickest film (189 nm) shows the typical MR of a Kondo lattice system, with an increasingly negative MR as the temperature is lowered, followed by a minimum and an increase of the MR that is due to the onset of coherence and the heavy-fermion (HF) state. In the thinnest films, the MR effect is much weaker, and there is no minimum, proving again that the coherent state is suppressed.

To summarize our experimental findings, we observe a dramatic suppression of the heavy-fermion state in the ground state of $\mathrm{CeCu}_{6}$ as the thickness of the films is reduced. Whereas the residual resistivity $\rho_{0}$ and the XRD measurements prove that the intrinsic disorder is not changed as the films are made thinner, characteristic signatures of the heavy-fermion ground state such as the high Fermi-liquid coefficient $A$ and the sharp minimum in the MR are reduced, and even completely suppressed in our thinnest films. As mentioned previously, the quality of the thin films was carefully analyzed, so that external effects such as preferred orientation changes, the grain structure or oxygen contamination can, as far as we can determine, be excluded as possible explanations for the observed thickness dependence. This striking effect is therefore an intrinsic property of $\mathrm{CeCu}_{6}$, and we will now consider possible scenarios for this thickness dependence of the correlated state.

\section{DISCUSSION}

As we have seen, the most common model used to describe heavy-fermion systems is that of the Kondo lattice. Let us first take this model as a starting point in our discussion and see whether reducing the sample dimensions can lead to our results. We can approach this in two ways: The single impurity Kondo scattering is affected by a reduction of the size of the samples (scenario I), or the Kondo temperature itself is size dependent (scenario II), affecting the electron-electron interactions leading to the coherent state in the Kondo lattice.

Finally, in scenario III, we will also put forward an alternative way of looking at $\mathrm{CeCu}_{6}$, based on the fact that this system lies close to a quantum critical point.

Let us first remark that it is not simply the coherence that is lost when the thickness is reduced. If that were the case, $\mathrm{CeCu}_{6}$ would just cross over from a periodic Kondo system to a dense independent-impurity Kondo system. The resistivity would increase down to zero temperature, with no change at high temperatures, leading to a strong increase of the residual resistivity. Also the magnitude of the MR would not change at the minimum, ${ }^{18}$ as opposed to the large suppression we observe for thinner films.

\section{A. Size dependence of single-impurity Kondo scattering}

It is generally accepted by now that the original picture of a "Kondo cloud" with finite spatial extension of spinpolarized electrons screening the magnetic moment of the Kondo impurity ${ }^{19}$ is not correct. This follows both from theoretical considerations ${ }^{20}$ and experimental results. ${ }^{22,23,25}$ However, a number of experimental observations were made on the size dependence of the Kondo effect, mostly using thin films or thin wires of very dilute Kondo alloys, e.g., $\mathrm{AuFe}^{22,23,26-28}$ or $\mathrm{CuCr}^{29,30}$ No clear picture emerges from these experimental results, since in some cases a size effect is indeed observed ${ }^{22,26-28,30}$ and in other cases not. ${ }^{23,29}$ What these results have in common though, is that the Kondo temperature appears to be constant. The size effect on the transport properties, if any, shows a reduction of the Kondo scattering term in thinner films or wires-scenario $\mathbf{I}$.

Two different theoretical models have been put forward to explain these results. The first, by Újsághy et al. ${ }^{31}$ considers the effect of spin-orbit interaction of the conduction electrons with the magnetic impurity. This interaction induces an impurity-spin anisotropy close to the surface of the sample, which reduces the effective thickness contributing to the Kondo scattering of the conduction electrons. While this may explain the observed reduction of the logarithmic slope of the resistivity of $\mathrm{CeCu}_{6}$ films as a function of temperature at high temperatures, such a scenario cannot explain our observation that at low temperatures, the Fermi-liquid coefficient is completely suppressed in films of $10 \mathrm{~nm}$ thickness. The second model, by Martin et al. ${ }^{32}$ describes how the interplay between spin scattering (Kondo) and weak localization (disorder) can modify the coefficient of the $\ln T$ dependence of the resistivity. However, their theory only applies in the very dilute case, in which normal impurity scattering leading to 
weak localization dominates over spin scattering. $\mathrm{CeCu}_{6}$ obviously does not belong to this category.

\section{B. Size dependence of the Kondo temperature}

Within the Kondo lattice model for heavy fermions, the Kondo temperature $T_{\mathrm{K}}$ is the fundamental energy scale of the system. Other commonly used energy scales, such as the coherence temperature or the Ruderman-Kittel-KasuyaYosida (RKKY) temperature, can be directly related to $T_{\mathrm{K}}$. Returning to the analogy with dilute Kondo systems, let us consider a thickness-dependent Kondo temperature as a possible cause of our results-scenario II. From Fermi-liquid theory, $A$ is proportional to $N\left(E_{\mathrm{F}}\right)^{2}$, the density of states (DOS) at the Fermi level squared. Our measurements thus show that the DOS at the Fermi level is reduced in thinner films. The Kondo temperature is related to the DOS at the Fermi level via $T_{\mathrm{K}} \propto 1 / N\left(E_{\mathrm{F}}\right)$ provided the Kondo interaction is much greater than the RKKY interaction, ${ }^{33}$ i.e., $J N\left(E_{\mathrm{F}}\right)$ $\gg 1$ with $J$ the magnetic coupling. An increase of the Kondo temperature $T_{\mathrm{K}}$ with decreasing thickness is in qualitative agreement with our results: An increased $T_{\mathrm{K}}$ reduces the magnetic interactions at low temperature and therefore suppresses the heavy Fermi-liquid state.

Unfortunately, transport measurements do not allow us to determine unambiguously the Kondo temperature in our system. The maximum in the resistivity $T_{\max }$ results from the onset of coherence in the heavy Fermi-liquid state and is therefore not a good measure for $T_{\mathrm{K}}$. The MR results can provide better information about the Kondo temperature. The calculations by Kawakami and Okiji ${ }^{18}$ show that for decreasing $H / T_{\mathrm{K}}$, the amplitude of the MR decreases. The change of amplitude in the MR of a factor of 5 as we observe in Fig. 4 can be explained by an increase of $T_{\mathrm{K}}$ by about a factor of 4. As is the case for $T_{\max }$, we cannot use the position of the minimum of the MR as a measure for $T_{\mathrm{K}}$, since we cross over from a Kondo lattice to an independent Kondo impurity system.

To explain the observed size dependence of Kondo scattering in point-contact experiments also a variation of the Kondo temperature with size was put forward. ${ }^{24}$ The theoretical explanation, given by Zaránd and Udvardi, ${ }^{21}$ is based not on a decrease of the DOS but rather on fluctuations of the local DOS near the surface of the point contact. For very small point contacts, the contribution of strong fluctuations of the local DOS near the surface of the point contact leads to the observation of an enhanced "effective" Kondo temperature. The smaller the value of the bulk Kondo temperature, the larger the enhancement in small point contacts. We do not believe that such an explanation can hold for our thin films of $\mathrm{CeCu}_{6}$ : First, the effects we observe occur at very large film thickness as compared to the length scales over which one may expect fluctuations of the DOS. ${ }^{21}$ Second, the Kondo temperature of the compounds that were studied in the point contact experiments were one to two orders of magnitude smaller than that of $\mathrm{CeCu}_{6}$, which has a bulk Kondo temperature of the order of $1 \mathrm{~K}$.

Whether scenario II can form the basis for explaining our results is highly debatable. This scenario is based on a com-

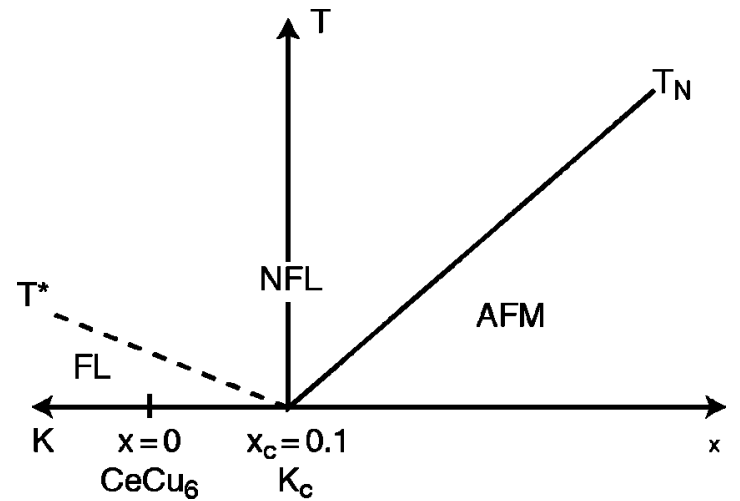

FIG. 5. Schematic phase diagram of the doped system $\mathrm{CeCu}_{6-x} \mathrm{Au}_{x}$ that undergoes a QPT at $x=0.1 . K$ is the coupling parameter measuring the strength of the quantum fluctuations. NFL stands for non-Fermi liquid, AFM stands for antiferromagnetic.

parison with a dilute Kondo model, with all its shortcomings when applying it to a Kondo lattice system: One of them lies in the assumption of no residual interactions or magnetic ordering appearing on the periodic Ce lattice. Indeed, even for large $T_{\mathrm{K}}$, interactions and antiferromagnetic order do remain a possibility at low temperatures and thereby the transport properties would be modified accordingly. ${ }^{13}$

\section{Dimensional crossover due to quantum criticality}

In order to get a deeper physical feeling for the observed effects, let us take a step back, and attempt to describe $\mathrm{CeCu}_{6}$ in a more general manner, our scenario III. We already mentioned that $\mathrm{CeCu}_{6}$ lies very close to a magnetic instability. Whether or not it does actually order antiferromagnetically at very low temperatures is still debatable, ${ }^{34}$ but there is not much needed to trigger such a transition: doping the system with gold leads to an antiferromagnetic (AFM) ground state for $\mathrm{CeCu}_{6-x} \mathrm{Au}_{x}$ with $x \geqslant 0.1$ (Fig. 5). It is now believed that the non-Fermi-liquid behavior observed at $x$ $=0.1$ arises from the QCP at that doping concentration. The general picture is the following (see Fig. 5): When describing the thermodynamics of a quantum system in $D$ dimensions, the expression for its partition function maps onto a classical partition function for a system with $D+1$ dimensions; the extra dimension is finite in extent and has units of time ${ }^{35}$ $\hbar / k_{\mathrm{B}} T$. At $T=0$, the quantum system undergoes a quantum phase transition (QPT) when the strength $K$ of the quantum fluctuations is changed and becomes equal to a critical value $K_{\mathrm{c}}$ : These fluctuations play the role of the thermal fluctuations in the classical system.

Therefore, using our knowledge of critical phenomena, a spatial correlation length $\xi$ diverges as $K \rightarrow K_{\mathrm{c}}$ with a critical exponent $\nu$. Similarly, there must be a correlation "length" $\xi_{\tau}$ in temporal direction, which may have a different critical behavior than $\xi$ because of the possibly different nature of the correlations in space and in time, respectively. Quite generally, the relation between $\xi$ and $\xi_{\tau}$ is governed by the dynamical exponent $z, \xi_{\tau} \sim \xi^{z}$. The effect of finite temperatures is to cut off the low-frequency fluctuations via $k_{\mathrm{B}} T \sim \hbar / \tau$. The characteristic temperature $T^{*}$ of the system is the tem- 
perature at which the cutoff frequency $1 / \tau$ is equal to the coherence frequency $1 / \xi_{\tau}$. This temperature marks a crossover with increasing temperature from a $(D+1)$ - to a $D$-dimensional system. Now, when the thickness of the films is decreased, the spatial correlation length $\xi$ is cut off at the thickness $d$ of the film for sufficiently small $d$. This leads to an effective increase of the coherence frequency, since now $\xi_{\tau} \sim d^{z}$. For sufficiently small $d$, the coherence "length" in temporal direction is now directly related to the thickness of the films, and therefore also the characteristic temperature $T^{*}$ is related to $d$ for sufficiently small $d$,

$$
T^{*} \propto d^{-z}
$$

We have seen that it is difficult to determine the temperature scale of our system based on transport measurements only. We believe that the Fermi-liquid coefficient $A$ is the most physical quantity that we can extract. Without requiring any of the concepts of Kondo theory, we can still relate $A$ to $T^{*}$. We know from Landau Fermi-liquid theory that $A$ $\propto N\left(E_{\mathrm{F}}\right)^{2}$. Dimensional analysis requires that $T^{*} \propto 1 / N\left(E_{\mathrm{F}}\right)$. Our experiments suggest a linear relationship between $A$ and the film thickness $d$. Putting these results in Eq. (1), we obtain $z=0.5$.

To explain our results within the framework of quantum criticality, we sketched in Fig. 5 a phase diagram where $d^{-1}$ is put on the horizontal axis, which normally measures the strength of the fluctuations. For, by decreasing the thickness of the films, we are increasing the characteristic temperature $T^{*}$ thus moving away from the QCP. This suggests that moving away from the Neél ordered state enhances the quantum spin fluctuations and therefore suppresses the heavy-fermion state, especially with a dimensionality reduction. In the phase diagram, this is equivalent to moving towards the left on the horizontal axis, or to stretching the $T$ axis and thereby pulling up the line describing $T^{*}$. We do not observe a significant increase of the residual resistivity due to this enhancement of the quantum fluctuations. The relationship between $A$ and $T^{*}$ was recently confirmed by measurements by Pfleiderer et al. ${ }^{36}$ They find that $A$ tends to diverge as $T^{*}$ becomes zero at the QCP, proving that heavy-fermion behavior is enhanced as one approaches the QCP. The value of the dynamical exponent $z=0.5$ is quite unusual, since this would mean that the spatial correlation length diverges faster than the temporal correlation length as the QCP is approached. The expected value of $z$ for an antiferromagnetic system is 2 , and is confirmed by the non-Fermi-liquid (NFL) behavior at the critical point, assuming two-dimensional AFM fluctuations. ${ }^{37,38}$ We therefore wish to stress here that the arguments we used to determine $z$ were merely qualitative, and that in order to confirm this picture of the HF system $\mathrm{CeCu}_{6}$, measurements of thermodynamic quantities such as the specific heat or the susceptibility in these thin films would be more appropriate. They are unfortunately very difficult to perform in view of the small available volume in thin films.

A length scale $\xi$ of $10 \mathrm{~nm}$ might seem very large at first sight. However, we are dealing with particularly "slow" quasiparticles, with a typical velocity $v_{\mathrm{F}}$ that is a factor 1000 lower than free electrons in normal metals. Also we have a system with a low characteristic energy scale $k_{\mathrm{B}} T^{*}$ of about $1 \mathrm{~K}$, which can be estimated, e.g., from the temperature where the coherent state becomes important. This characteristic energy corresponds to a time scale $\xi_{\tau}=10^{-11} \mathrm{~s}$ leading indeed to a characteristic length of $v_{\mathrm{F}} \xi_{\tau}=10 \mathrm{~nm}$. It is actually this very low value of $T^{*}$ that is believed to make $\mathrm{CeCu}_{6}$ and other so-called "Kondo lattice" compounds heavy-fermion systems.

\section{CONCLUSIONS}

Our measurements on thin films of $\mathrm{CeCu}_{6}$ have exhibited a dramatic change of the ground-state properties upon approaching a thickness of the order of $10 \mathrm{~nm}$.

Because $\mathrm{CeCu}_{6}$ is usually described as a Kondo-lattice system, we have attempted to reconcile our results with the properties of a dilute Kondo system. A size effect of the Kondo effect itself (scenario I), as observed experimentally in dilute Kondo systems, does not account for the scaling of characteristic energies that we observe as a function of thickness $\left(A\right.$ and $\left.T_{\max }\right)$. The characteristic energy of the Kondo lattice model is the Kondo temperature $T_{\mathrm{K}}$, and a thickness dependence of $T_{\mathrm{K}}$ can qualitatively explain our results (scenario II). However, it is not clear whether this scenario also applies to a Kondo lattice, and why fluctuations of the DOS should occur at the relatively large length scale of around 10 nm.

The proximity of $\mathrm{CeCu}_{6}$ to a quantum critical point has led us to seek an explanation in terms of another description of $\mathrm{CeCu}_{6}$ (scenario III). The physics of a system near a QCP is governed by two correlation lengths, $\xi_{\tau}$ in the time direction and $\xi$ in spatial direction that diverge as one approaches the QCP. When, as is the case for $\mathrm{CeCu}_{6}$, the quantum fluctuations are so large that the system is not ordered at $T=0$, $\xi_{\tau}$ sets a characteristic temperature scale $T^{*}$ above which the system "loses" one dimension. Because $\xi_{\tau}$ and $\xi$ are coupled through the dynamical critical exponent $z$, an effective reduction of one spatial dimension below $\xi$ has the effect of increasing the characteristic temperature scale $T^{*}$. This leads on the one hand to a "stretching" of the temperature axis, i.e., a flattening of resistivity as a function of temperature. On the other hand, it leads to a lower DOS at the Fermi level, and hence to a weakening of the correlations (decrease of $A$ ). This scenario therefore provides a reasonable "starting point" for the length scale that was found in our experiments although we cannot explain the very small values of $A$ for the thinnest films and the residual resistivity does not reflect the increase of the quantum fluctuations.

\section{ACKNOWLEDGMENTS}

We wish to thank L. Qin and H. W. Zandbergen from the National Center for HREM in Delft, The Netherlands, for the HREM measurements on our films. Furthermore, we would like to acknowledge J. Zaanen for enlighting discussions on the theory of quantum phase transitions. This work was supported by the Dutch Foundation for Fundamental Research on Matter (FOM). 
${ }^{1}$ F. Steglich, U. Ahlheim, C.D. Bredl, C. Geibel, M. Lang, A. Loidl, and G. Sparn, in Frontiers in Solid State Science, edited by L.C. Gupta and M. S. Multani (World Scientific Publishing Company, New York, 1991), Vol. 1, p. 1.

${ }^{2}$ D.L. Cox and M.B. Maple, Phys. Today 48 (2), 32 (1995).

${ }^{3}$ Z. Fisk, D.W. Hess, C.J. Pethick, D. Pines, J.L. Smith, J.D. Thompson, and J.O. Willis, Science 239, 33 (1988).

${ }^{4}$ G.J. Nieuwenhuys, in Handbook of Magnetic Materials, edited by K.H.J.Buschow (Elsevier Science B.V., Amsterdam, Netherlands, 1995), Vol. 9, Chap. 1, p. 1.

${ }^{5}$ J.A. Mydosh, Z. Phys. B 103, 251 (1997).

${ }^{6}$ G. Aeppli and Z. Fisk, Comments Condens. Matter Phys. 16, 155 (1992).

${ }^{7}$ M.B. Maple, M.C. de Andrade, J. Herrmann, Y. Dalichaouch, D.A. Gajewski, C.L. Seaman, R. Chau, R. Movshovich, M.C. Aronson, and R. Osborn, J. Low Temp. Phys. 99, 223 (1995).

${ }^{8}$ E. Bauer, Adv. Phys. 40, 417 (1991).

${ }^{9}$ G.R. Stewart, Z. Fisk, and M.S. Wire, Phys. Rev. B 30, 482 (1984).

${ }^{10}$ H.G. Schlager, A. Schröder, M. Welsch, and H. von Löhneysen, J. Low Temp. Phys. 90, 181 (1993).

${ }^{11}$ H.R. Ott, H. Rudigier, Z. Fisk, J.O. Willis, and G.R. Stewart, Solid State Commun. 53, 235 (1985).

${ }^{12}$ A. Amato, D. Jaccard, J. Flouquet, F. Lapierre, J.L. Tholence, R.A. Fisher, S.E. Lacy, J.A. Olsen, and N.E. Phillips, J. Low Temp. Phys. 68, 371 (1987).

${ }^{13}$ Recently Tsujii et al. have measured the magnetic susceptibility and thermal expansion of single crystals of $\mathrm{CeCu}_{6}$ that indicate the occurence of antiferromagnetic order below $2 \mathrm{mK}$. Note that this transition does not fall on the line describing the AFM transition in the system doped with $\mathrm{Au}$, which extrapolates to zero at exactly $x=0.1$. H. Tsujii, E. Tananka, Y. Ode, T. Katoh, T. Mamiya, S. Araki, R. Settai, and Y. Ǒnuki, Phys. Rev. Lett. 84, 5407 (2000).

${ }^{14}$ H. von Löhneysen, J. Phys.: Condens. Matter 8, 9689 (1996).

${ }^{15}$ D. Groten, G.J.C. van Baarle, R.W.A. Hendrikx, J. Aarts, G.J. Nieuwenhuys, and J.A. Mydosh, Physica B 259, 30 (1999).

${ }^{16}$ S.P. Strong and A.J. Millis, Phys. Rev. B 50, 12611 (1994).

${ }^{17}$ G. Baym and C. Pethick, Landau Fermi-Liquid Theory (Wiley, New York, 1991).
${ }^{18}$ N. Kawakami and A. Okiji, J. Phys. Soc. Jpn. 55, 2114 (1986).

${ }^{19}$ J.E. Gubernatis, J.E. Hisch, and D.J. Scalapino, Phys. Rev. B 35, 8478 (1987).

${ }^{20}$ P.W. Anderson, G. Yuval, and D.R. Hamann, Phys. Rev. B 1, 4464 (1970).

${ }^{21}$ G. Zaránd and L. Udvardi, Phys. Rev. B 54, 7606 (1996).

${ }^{22}$ N. Giordano, Phys. Rev. B 53, 2487 (1996).

${ }^{23}$ V. Chandrasekhar, P. Santhanam, N.A. Penebre, R.A. Webb, H. Vloeberghs, C. Van Haesendonck, and Y. Bruynseraede, Phys. Rev. Lett. 72, 2053 (1994).

${ }^{24}$ I.K. Yanson, V.V. Fisun, R. Hesper, A.V. Khotkevich, J.M. Krans, J.A. Mydosh, and J.M.V. Ruitenbeek, Phys. Rev. Lett. 74, 302 (1995).

${ }^{25}$ D. Goldhaber-Gordon, J. Göres, M.A. Kastner, H. Shtrikman, D. Mahalu, and U. Meirav, Phys. Rev. Lett. 81, 5225 (1998).

${ }^{26}$ G. Chen and N. Giordano, Phys. Rev. Lett. 66, 209 (1991).

${ }^{27}$ M.A. Blachly and N. Giordano, Phys. Rev. B 51, 12537 (1995).

${ }^{28}$ V. Chandrasekhar, P. Santhanam, N.A. Penebre, R.A. Webb, H. Vloeberghs, C. Van Haesendonck, and Y. Bruynseraede, Physica B 194, 1053 (1994).

${ }^{29}$ C. van Haesendonck, J. Vranken, and Y. Bruynseraede, Phys. Rev. Lett. 58, 1968 (1987).

${ }^{30}$ J.F. DiTusa, K. Lin, M. Park, M.S. Isaacson, and J.M. Parpia, Phys. Rev. Lett. 68, 678 (1992).

${ }^{31}$ O. Újsághy, A. Zawadowski, and B.L. Gyorffy, Phys. Rev. Lett. 76, 2378 (1996).

${ }^{32}$ I. Martin, Y. Wan, and P. Phillips, Phys. Rev. Lett. 78, 114 (1997).

${ }^{33} \mathrm{~S}$. Doniach, in Valence Instabilities and Related Narrow-Band Instabilities, edited by R. D. Parks (Plenum Press, New York, 1993), p. 435.

${ }^{34}$ E.A. Schuberth, J. Schupp, R. Freese, and K. Andres, Phys. Rev. B 51, 12892 (1995).

${ }^{35}$ S.L. Sondhi, S.M. Girvin, J.P. Carini, and D. Shahar, Rev. Mod. Phys. 69, 315 (1997).

${ }^{36}$ C. Pfleiderer, B. Will, O. Stockert, and H. von Löhneysen, Physica B 281, 363 (2000).

${ }^{37}$ A. Rosch, A. Schröder, O. Stockert, and H. von Löhneysen, Phys. Rev. Lett. 79, 159 (1997).

${ }^{38}$ O. Stockert, H. von Löhneysen, A. Rosch, N. Pyka, and M. Loewenhaupt, Phys. Rev. Lett. 80, 5627 (1998). 